\title{
Variations
}

Variations

Revue internationale de théorie critique

$23 \mid 2020$

Pour une gauche érotique

\section{Autonomie et émancipation : la Théorie critique à la lumière de l'Aufklärung kantienne}

\section{Emmanuel Chaput}

\section{(2) OpenEdition}

Journals

Édition électronique

URL : http://journals.openedition.org/variations/1558

DOI : 10.4000/variations. 1558

ISSN : 1968-3960

Éditeur

Les amis de Variations

\section{Référence électronique}

Emmanuel Chaput, « Autonomie et émancipation : la Théorie critique à la lumière de l'Aufklärung kantienne », Variations [En ligne], 23 | 2020, mis en ligne le 01 septembre 2020, consulté le 07 septembre 2020. URL : http://journals.openedition.org/variations/1558 ; DOI : https://doi.org/ 10.4000/variations. 1558

Ce document a été généré automatiquement le 7 septembre 2020.

Les ami•e•s de Variations 


\title{
Autonomie et émancipation : la Théorie critique à la lumière de l' Aufklärung kantienne
}

\author{
Emmanuel Chaput
}

1 Il n'est pas aisé de démêler les fils du rapport complexe et souvent trouble qu'entretiennent les principaux auteurs de l'École de Francfort avec les Lumières kantiennes. Tant la première que la seconde génération ont tour à tour fait la critique d'Emmanuel Kant, tout en se réappropriant, à tout le moins implicitement, une partie du projet kantien. Ce double rapport est peut-être le plus manifeste chez Jürgen Habermas. Toute son entreprise philosophique, tant dans le domaine épistémologique (Habermas, $1987: 61$ ) que pratique (Habermas, 1992 : 70-71; Baynes, $2004: 194)$ ), est en effet à inscrire dans une visée émancipatrice qui constitue précisément, à ses yeux, l'essence même de l'Aufklärung kantienne.

\section{Habermas ou la puissance émancipatrice des lumières contre l'autoritarisme philosophique}

Dans la section de L'Espace public intitulée « La publicité médiatrice de la politique et de la morale » que Habermas consacre à Kant, celui-ci écrit en effet : «L'émancipation par rapport à cette immaturité dont on porte soi-même la responsabilité, c'est précisément cela qui s'appelle Aufklärung " (Habermas, 1986 : 114 [nous soulignons]). Habermas fait donc sien le projet des Lumières kantiennes, certes en le radicalisant, en inscrivant tout son travail de savant (Gelehrter) ${ }^{1}$ dans une perspective émancipatrice. "Comme toujours, souligne à juste titre Claude Piché, c'est la tâche émancipatrice de l'Aufklärung qui réquisitionne les services de la théorie, qui en dicte la teneur et les paramètres " (Piché, 1995 : 217). L'Aufklärung, surtout dans sa forme kantienne fondée sur " un usage public de sa raison » (Kant, 1985 : 211) demeure, comme le note encore Piché, « centrale pour toute la pensée de Habermas» (Piché 1995 : 217). Et pourtant, ce vestige des Lumières kantiennes n'a pas empêché Habermas de vertement critiquer Kant. Et 
malgré tout, on peut néanmoins déceler derrière certaines de ces critiques un motif foncièrement kantien, comme si Habermas pensait à la fois avec et contre Kant.

3 On voit ainsi Habermas reprocher à Kant d'attribuer indûment une fonction d' assignation (Platzanweiser) voire de juge (Richter) à la raison philosophique (Piché, 1995 : 194-95). Kant « confère à la philosophie, d'après Habermas, le rôle de juge suprême, rôle qui s'étend d'ailleurs à l'ensemble de la culture » (Habermas, $1997: 24-25)$. C'est elle qui, en définitive, doit légiférer tant dans le domaine des sciences, que de la morale et de l'esthétique. Dans son texte sur "L'héritage néo-kantien de Jürgen Habermas », Piché a bien raison de souligner l'inexactitude d'une telle critique :

Il s'avère [...] après examen, que bien que les trois Critiques exposent respectivement les conditions de possibilité du sens commun esthétique, de l'entendement commun moral ainsi que de l'entendement commun cognitif, il ne s'ensuit pas pour autant que le discours transcendantal impose quoi que ce soit à son objet. (Piché, 1995 : 202)

4 Il importe cependant de souligner qu'au final, on retrouve au cœur de cette critique certes philologiquement inadéquate ou injuste encore un motif essentiellement kantien. Habermas écrit :

Avec la science moderne, avec le droit positif et les éthiques profanes guidées par des principes, avec un art devenu autonome et l'institutionnalisation de la critique d'art, ce sont trois moments de la raison qui se sont cristallisés d'eux-mêmes, sans aucune intervention de la philosophie. (Habermas, 1997 : 38)

5 C'est donc par souci de préserver l'autonomie et de reconnaître le passage d'un état de minorité à celui d'une majorité qu'Habermas défend les raisons scientifiques, morales et esthétiques vis-à-vis de l'autorité extérieure de la raison philosophique. On retrouve ainsi les grands thèmes bien connus des Lumières kantiennes :

Les lumières se définissent comme la sortie de l'homme hors de l'état de minorité [...] La minorité est l'incapacité de se servir de son entendement sans être dirigé par un autre [...] Sapere aude! Aie le courage de te servir de ton propre entendement ! Voilà la devise des lumières. (Kant, $1985: 210)$

Dans le cas présent, c'est comme si c'était les sciences, l'art et la morale elle-même qui s'émancipaient, qui définissaient leurs propres règles trop longtemps déterminées de l'extérieur, de manière hétéronome, soit par la religion ou la philosophie. On peut donc dire que le reproche d'« autoritarisme philosophique " porté par Habermas à l'endroit de Kant s'inscrit dans la perspective émancipatrice ouverte par les Lumières kantiennes elles-mêmes. Nous verrons que, d'une certaine manière, ce motif revient constamment chez les penseurs de la première génération de l'école de Francfort.

$7 \mathrm{Au}$ cœur de leur critique des Lumières, de la raison et du progrès, on retrouve encore le «noyau rationnel » - pour reprendre l'expression consacrée par Karl Marx (Marx, 2009 : 18) - non seulement de la dialectique hégélienne et du matérialisme historique, mais encore des Lumières kantiennes. On a souvent en effet discuté des thèses de l'École de Francfort à l'aune de l'hégéliano-marxisme et de la pensée dialectique. Mais l'influence kantienne n'est pas moins importante en un sens. Quelqu'un comme Pierre Zima ira même jusqu'à dire que la Théorie critique opère dans les faits un retour à Kant - il parlera même en fait d'une "régression" par rapport à Hegel et Marx (Zima, $1974: 124)$.

8 Si l'on fait en un sens exception de Herbert Marcuse (Marcuse, 1968; Marcuse, 1991 ; Assoun, 2012: 37-38), il est vrai que les auteurs les plus importants de la première génération de l'École de Francfort tels que Max Horkheimer et Theodor 
Adorno s'opposent largement à la logique de l'identité hégélienne, à l'idée d'un système totalisant. "Le tout est le non-vrai» écrit Adorno (Adorno, 2008: 64), alors que Horkheimer écrit pour sa part dans "Hegel et le problème de la métaphysique " (1932): "Dans cette philosophie les différences et les tensions ne peuvent être réinterprétées comme "contradictions" qu'autant qu'elles sont a priori conçues comme pensées du sujet, embrassant tout et identique à tout » (Horkheimer, 1980 : 140-41). Et bien que l'Institut für Sozialforschung fut dès sa naissance baignée dans les eaux baptismales du marxisme, l'expérience de l'échec révolutionnaire en Allemagne, la montée du fascisme, le travestissement du marxisme soviétique et l'intégration progressive des grands syndicats à la logique du capital ont grandement contribué au pessimisme politique des penseurs de l'École de Francfort, à l'exception peut-être encore une fois et dans une certaine mesure seulement, de Marcuse.

L'essentiel des travaux de l'École de Francfort porte en effet sur les multiples mécanismes d'intégration, les nouvelles logiques de domination et les différentes figures de la société "sur-répressive» (Marcuse, 1971b: 44). Certes, l'impératif d'émancipation qui comme nous l'avons vu constitue le noyau des Lumières kantiennes selon Habermas est déjà bien présent dès les premières formulations de la Théorie critique. Ainsi, dans son écrit programmatique "Théorie traditionnelle et théorie critique » (1937), Horkheimer écrit en effet : «la théorie critique n'a pour elle aucune autre instance spécifique que l'intérêt des masses à la suppression de l'injustice sociale » (Horkheimer, $1996: 80$ ). Cependant avec l'avènement des sociétés totalitaires, le développement du capitalisme avancé, et l'échec des révolutions socialistesdémocratiques, la Théorie critique articule d'une certaine manière un repli par rapport à la perspective politico-institutionnelle, voir révolutionnaire de l'émancipation vers une perspective éthico-esthétique devant préserver l'autonomie à la fois du sujet moral et réflexif et des œuvres d'art authentiques (Adorno). Comme l'écrit Zima: «Face à l'intégration de la classe révolutionnaire, il s'agit de sauver ce qui reste de l'individualisme, de l'autonomie, de la capacité critique » (Zima, 1974 : 24 ; 18-19). C'est pourquoi ce dernier parlera d'une « régression » par rapport à Hegel et Marx vers Kant. Plutôt que de penser la rationalité du présent avec G.W.F. Hegel ou la praxis révolutionnaire devant nous faire basculer du côté de cette société rationnelle à venir qu'est le communisme de Marx, il s'agit de préserver les vestiges de la pensée critique, l'héritage des lumières qui semble constamment trahies par le développement des Lumières elles-mêmes. Il s'agit de préserver l'individu moral et réflexif de la logique " ensembliste-identitaire " ou " ensidique " pour parler comme Cornélius Castoriadis (Castoriadis, 1999) qui fait de l'individu un être subordonné à un tout et un simple numéro dans une série de chiffres indifférenciés. C'est pourquoi, selon Zima: «La régression à Kant effectuée par la Théorie critique signifie qu'elle adopte une attitude éthique, négative [...] à l'égard de la société contemporaine" (Zima, 1974: 124). Comprise comme le lieu de la réification et de l'asservissement, la société, le tout, est le non-vrai, duquel il faut préserver l'individu. Ce qui constitue donc pour les penseurs de la Théorie critique la forme aboutie de l'émancipation, c'est d'abord et avant tout, par conséquent, l'autonomie. Le moyen de garantir celle-ci sera l'attitude critique, la vigilance incessante contre le dogmatisme, même et peut-être surtout lorsqu'il revêt les atours du progrès et des Lumières. "C'est à l'existence de l'attitude critique, écrit Horkheimer, qu'est aujourd'hui suspendu l'avenir de l'humanité » (Horkheimer, 1996 : 81). Autonomie, attitude critique, l'individu considéré comme fin et jamais comme 
moyen, on le voit clairement, la Théorie est critique en un sens résolument kantien. Mais ce « retour à Kant » ne s'empêchera pas d'être tout aussi critique de Kant.

Et pourtant, on sent clairement l'inspiration kantienne de la Théorie critique de l'École de Francfort, et ce dès les Studien über Autorität und Familie, qui constitue le premier rapport publié par l'Institut (Assoun, 2012: 67; Wiggerhaus, 1993: 143). Les thèmes mêmes de l'enquête, et au premier chef le rapport à l'autorité, constituent en effet des thèmes centraux de la philosophie pratique de Kant. Dès l'introduction générale de Horkheimer, on retrouve la même position ambivalente à l'égard de l'autorité que l'on retrouve dans le Qu'est-ce que les Lumières? (1784) de Kant. Kant souligne en effet dans cet écrit :

il serait très dangereux qu'un officier, qui a reçu un ordre de ses supérieurs, se mît à raisonner dans le service sur l'opportunité ou l'utilité de cet ordre ; il doit obéir. Mais on ne peut pas légitimement lui interdire de faire, en tant que savant, des remarques sur les erreurs touchant le service militaire et les soumettre à son public afin qu'il les juge. (Kant, $1985: 212$ )

11 C'est la fameuse distinction entre l'usage public et privé de notre raison sur laquelle nous reviendrons. J'aimerais simplement souligner que pour Kant autant que pour la Théorie critique, ce n'est pas l'autorité en soi, qui doit faire l'objet de critique, mais son caractère superfétatoire. Critique ici, encore une fois, signifie discernement (du grec

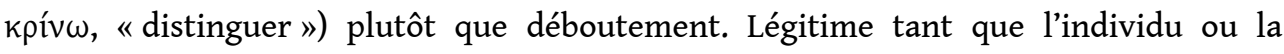
culture se trouve, non par sa propre faute, mais par le fruit de conditions objectives qui le dépassent, dans un état de minorité, l'autorité doit s'effacer là où elle devient superflue du fait du progrès humain. Ainsi, Horkheimer écrit :

Pendant des périodes entières il était de l'intérêt des individus dominés de se soumettre, comme l'enfant a intérêt à une bonne éducation. C'était une condition nécessaire pour que les aptitudes des hommes puissent se développer. Mais même en tenant compte des époques où ce rapport de dépendance était sans conteste adapté à l'état des forces humaines et de leurs instruments, il a été lié jusqu'à nos jours à des renoncements imposés aux sujets [...] l'acceptation par les sujets des rapports de dépendance existants [...] ne signifiait pas seulement que leur impuissance devait être éternisée sur le plan matériel, mais aussi sur le plan spirituel; elle freinait donc, d'une manière générale, l'évolution de l'humanité. (Horkheimer, $1996: 251$ )

Si l'autorité peut jouer un rôle légitime dans l'histoire du développement humain tant d'un point de vue onto- que phylogénétique, elle pèche par excès en extrapolant ou en éternisant comme le dit Horkheimer sa fonction au-delà des besoins de ses sujets. Au contraire, comme le souligne Kant dans son apologie de Frédéric, le rôle du gouvernement, à l'ère où ce n'est plus par « manque d'entendement» (Kant, 1985 : 209) que l'homme est maintenu dans son état de minorité, est justement d'offrir les conditions objectives pour l'expression publique de cette intelligence. Frédéric est, selon Kant, «le premier à avoir affranchi le genre humain de la minorité, du moins pour ce qui relève du gouvernement, le premier à avoir laissé chacun libre de se servir de sa propre raison dans toutes les questions touchant la conscience» (Kant, 1985 : 216).

13 Deux choses sont importantes à retenir de ce passage. Premièrement, il faut souligner que l'affranchissement venant de l'autorité même du gouvernement reste quoique nécessaire, partiel. Il garantit la liberté de pensée aux membres de la société civile, mais ne peut en aucun cas se suppléer au «manque de résolution et de courage » qu'il faut pour user de son propre entendement «sans être dirigé par un autre» (Kant, 
1985 : 209). En définitive, ce n'est que le sujet moral, l'individu qui peut s'émanciper de son état de minorité. Nul ne peut le faire à notre place (Kant, 1985 : 209 ; Horkheimer, 1996 : 259 ; Marcuse, 1971a : 51).

14 Le second aspect à souligner dans ce passage est la délimitation de la liberté d'usage de la raison au domaine de la conscience. Or, c'est là l'impasse des Lumières allemande selon la Théorie critique : « on s'accommodait de l'oppression extérieure en croyant à la liberté enfermée dans les cœurs, et l'on soulignait d'autant plus l'indépendance de la personne que la personne réelle était plus asservie » (Horkheimer, $1996: 260)^{2}$.

15 La Théorie critique reproche ainsi à l'Aufklärung de cantonner son entreprise à la sphère privée de la conscience, sans jamais formuler une critique des formes d'oppressions publiques, sociales et économiques (Adorno, 2001 : 63). Au contraire, la liberté de conscience des individus privés apparaît objectivement comme l'une des conditions de possibilités de la forme répressive de la société moderne (Marcuse, 1971a : 71-72). Or, on pourrait penser que Kant, dans la mesure où sa conception des Lumières se fonde entièrement sur l'usage public de la raison, serait cependant épargné par cette critique. Mais il n'en est rien, comme nous le verrons.

16 Il faut cependant se tourner vers la contribution de Marcuse aux Studien über Autorität und Familie, pour comprendre en quoi Kant tombe lui aussi dans le subjectivisme qui constitue, plus largement, le travers de toutes les Lumières allemandes pour l'École de Francfort ${ }^{3}$.

\section{Marcuse, un Aufklärer contre les lumières ${ }^{4}$}

17 En posant les Lumières du côté de l'usage public de la raison et le devoir d'obéissance du côté de l'usage privé "sans pour autant empêcher sensiblement le progrès des lumières » (Kant, 1985 : 211), Kant semble prendre, pour Marcuse, le contrepied « de la solution luthérienne, qui avait soumis absolument la liberté publique à l'autorité séculière, tout en préservant absolument la liberté "intérieure" de la personne privée » (Marcuse, 1971a : 53). Mais comme le souligne Marcuse, il faut d'abord déterminer « ce que Kant entend par usage “public" et usage "privé” de la liberté » (Marcuse, 1971a : 53).

18 Or, l'usage public de la raison est défini par Kant comme «celui que l'on en fait comme savant devant l'ensemble du public qui lit » (Kant, 1985 : 211). L'usage privé au contraire est « celui qu'on a le droit de faire de sa raison dans tel ou tel poste civil, ou fonction, qui nous est confié » (Kant, 1985: 211). Comme le souligne à juste titre Marcuse : "Le "privé" est maintenant la "fonction" civile dont le titulaire doit subordonner sa raison à l'intérêt du "disciplinement" social » (Marcuse, 1971a: 53). À l'instar de Luther, la soumission au pouvoir séculier, à l'autorité ecclésiastique et à l'employeur demeure donc, dans l'exercice de nos fonctions, absolue. C'est en tant que savant, on dirait aujourd'hui en tant que chercheur indépendant, qu'on peut exercer notre liberté d'opinion et de penser. L'usage public de la raison prend ainsi la forme d'un débat fondé au pire sur l'opinion, au mieux sur l'expertise entraînant possiblement une réforme que dans la mesure où le pouvoir politique doit demeurer, pour Kant, à l'écoute de la volonté générale qui en fonde le pouvoir même. L'espace public ou la société civile comme lectorat devient ainsi la médiation entre les avis éclairés des savants et la possibilité de réformes sociales. 
19 Mais Marcuse va trop loin lorsqu'il affirme « que la liberté publique, qui ne subit pas de limitation, se trouve refoulée dans la dimension de la science pure et du "public des lecteurs" " (Marcuse, 1971a: 53-54). Comme nous l'avons vu, loin d'être limitée à la science pure, la raison éclairée peut s'intéresser à tout objet susceptible d'intérêt public. En donnant l'exemple de l'officier, il va sans dire que Kant prend le titre de savant en un sens large, auquel on pourrait peut-être aujourd'hui suppléer celui d'expert? En refoulant l'usage public de la raison du côté des sciences pures, Marcuse veut suggérer qu'au final les Lumières kantiennes ne s'expriment que dans une république des lettres de plus en plus "séparée de l'action publique et libre, de la pratique sociale réelle» (Marcuse, 1971a : 54). Tel n'est peut-être pas tout à fait le cas cependant.

20 Mais la difficulté demeure, et à ce titre Marcuse n'a pas tout à fait tort, d'articuler chez Kant la soumission dans la sphère des fonctions sociales avec la liberté de conscience publique. Il semble que d'un côté se situe la conscience du sujet moral autonome et de l'autre la sphère du droit responsable de la gestion des rapports entre les sujets et leurs propriétés. D'un côté, il y aurait la sphère de l'intériorité, c'est-à-dire la morale. De l'autre, la sphère de l'extériorité, le droit. Or, pour Marcuse, il semble bien y avoir une désarticulation entre les deux : "Pour Kant, écrit Marcuse, le droit en général n’a pas d'autre objet que "ce qui est extérieur dans les actions". La personne en tant que sujet "moral", en tant que lieu de la liberté transcendantale n'est pas en jeu dans la dimension du droit, autrement dit dans l'ordre de la société civile " (Marcuse, 1971a : 55-56). Dans l'ordre du droit, la personne doit se soumettre aux impératifs de la loi, aux us et coutumes qui peuvent apparaitre comme extérieurs à soi. Le droit serait-il ainsi la sphère de l'hétéronomie? Le pouvoir auquel doit obéissance la personne comme citoyen est-il fondé sur une puissance étrangère à la personne comme sujet autonome et libre ? Ce n'est pas exactement le cas pour Kant, dans la mesure où le droit doit, à ses yeux, être fondé, en définitive, sur la libre auto-limitation volontaire des sujets à la sphère du social. « La non-liberté civile de l'individu sous l'autorité légale des instances sociales est censée s'accorder avec le concept fondamental de la personne essentiellement libre, en ce sens que cette non-liberté est conçue comme l'autolimitation originellement égale et réciproque de tous les individus» (Marcuse, 1971a : 61). Mais d'une certaine manière, pour Marcuse, c'est comme si l'auto-nomie marquant la liberté du sujet moral devenait, dans la sphère du droit, auto-subordination à l'hétéronomie sociale. On retrouve ainsi ici ce lieu commun de la Théorie critique consistant à voir la sphère sociale dans sa réalité présente comme le domaine de l'hétéronomie et de l'aliénation.

21 Or, d'une certaine manière, l'alternative de la Théorie critique reste en un sens très kantienne. Plutôt que de considérer la réalité sociale et sa dimension "sur-répressive " comme l'expression d'une auto-limitation libre du sujet moral, elle consistera à faire justement de ce sujet moral, critique, le lieu de la contestation des formes actuelles de l'oppression. En acceptant de subordonner la liberté de conscience à la sphère de l'activité civile, c'est un peu comme si chez Kant nous délimitions un lieu où l'individu pourrait effectivement servir de simple moyen.

22 Bien que la critique de Marcuse, tout comme celle de Habermas, ne rend pas complètement justice à la position kantienne, il importe encore une fois de souligner à quel point elle demeure tributaire des Lumières kantiennes dans son esprit. Encore une fois c'est avec et contre Kant que la Théorie critique articule sa position. Encore une 
fois, c'est à une radicalisation des principes kantiens que l'on assiste. Pour Marcuse en effet, l'autonomie du sujet moral ne peut jamais être subordonnée à sa fonction sociale. $\mathrm{Au}$ contraire, le non-vrai de la totalité sociale doit faire l'objet d'une critique sans complaisance d'un tel sujet libre. L'autonomie kantienne doit ainsi devenir une puissance de transformation sociale selon Marcuse: "La liberté transcendantale de l'homme, l'autonomie absolue de la personne rationnelle reste le principe suprême dans toutes les dimensions de la philosophie kantienne; sur ce point il n'existe aucun compromis. Le fait que cette liberté ne devienne pas un pouvoir social pratique, que la liberté de pensée n'inclue pas la "liberté d'action" a son fondement dans l'ordre social où Kant a élaboré sa philosophie » (Marcuse, 1971a : 66-67). D’une certaine façon, Kant se trouve ainsi excusé, sinon innocenté. Il a poussé le principe des Lumières aussi loin qu'il lui était possible comme savant, dans le cadre social qui était le sien. Mais la Théorie critique pense dans un tout autre ordre social, où les alternatives existent, tant chez Marx que dans le socialisme démocratique.

23 Néanmoins, on voit à quel point, en un sens, la Théorie critique s'inscrit dans le prolongement de l'Aufklärung kantienne ${ }^{5}$. Certes, l'héritage marxiste fait en sorte que les modalités de l'émancipation sont désormais posées sur un plan plus proprement politique, mais l'École de Francfort reste néanmoins marquée par l'éthique kantienne et son refus de subordonner l'autonomie de l'individu moral à l'émancipation collective. Il s'agit d'articuler la critique de l'aliénation sociale avec celle de la conscience réflexive et morale du sujet. D'où la critique du révisionnisme néo-freudien en psychanalyse et du marxisme soviétique dans l'œuvre de Marcuse (Marcuse, 1963 ; Marcuse, 1971b; Benoist, 1970 :102-03), mais aussi des autres penseurs de la Théorie critique. Il s'agit de dénoncer toutes les formes de répression du sujet individuel y compris et surtout lorsque ces formes de répression prétendent à la libération du sujet qu'elles ne servent réellement qu'à asservir.

\section{Autonomie des individus libres ou autonomisation de la raison instrumentale}

Une telle critique est d'ailleurs au centre de la Dialektik der Aufklärung de Horkheimer et Adorno. Dans cet écrit séminal, ces derniers veulent en effet démontrer que le projet d'émancipation constitutif des Lumières se retourne contre lui-même et prend la forme de l'objet même de sa critique.

De tout temps, écrivent Horkheimer et Adorno, l'Aufklärung, au sens large de pensée en progrès, a eu pour but de libérer les hommes de la peur et de les rendre souverains. Mais la terre, entièrement "éclairée", resplendit sous le signe des calamités triomphant partout. Le programme de l'Aufklärung avait pour but de libérer le monde de la magie. Elle se proposait de détruire les mythes et d'apporter à l'imagination l'appui du savoir. (Horkheimer et Adorno, $1983: 21$ )

Et pourtant, elle finit par devenir elle-même une nouvelle forme mythique, celle du savoir éclairé, essentiellement technique et instrumental, se présentant comme l'unique source possible de bonheur et de libération. Ce faisant, la liberté devient ellemême une forme de domination, celle de la nature, du hasard et de l'irrationnel. Mais cette domination, cette suspicion face à ce qui ne semble pas être du penser dans toute la rigueur du terme équivaut à un repli unidimensionnel de la raison sur l'un de ses pôles constitutifs au détriment de sa multiplicité dialectique. « Une des caractéristiques 
de la rationalité a toujours été dès le début, sa tendance à s'autodétruire " (Horkheimer et Adorno, 1983: 19). Pour Adorno et Horkheimer, une telle autodestruction prend la forme d'un affaissement de la raison substantielle à sa dimension strictement instrumentale, dominatrice et destructrice : « L'intellect devient en effet l'instrument de la domination et de la maitrise de soi, que la philosophie bourgeoise a toujours cru à tort voir en lui » (Horkheimer et Adorno, 1983: 52; Bronner, 2011 : 55).

26 Cependant, une telle critique n'est pas à situer hors de la raison elle-même. Comme l'affirme Michel Ratté, la Théorie critique est encore à penser « en tant que projet de la raison » (Ratté, $2010: 231 ; 232)^{6}$. Au demeurant, Horkheimer et Adorno l'écrivent euxmêmes dans l'introduction à la Dialektik der Aufklärung:

Nous n'avons pas le moindre doute - et c'est là notre pétition de principe - que dans la société, la liberté est inséparable du penser éclairé. Mais nous croyons avoir tout aussi nettement reconnu que la notion même de ce penser, non moins que les formes historiques concrètes, les institutions de la société dans lesquelles il est imbriqué, contiennent déjà le germe de cette régression qui se vérifie partout de nos jours. Si la Raison n'entreprend pas un travail de réflexion sur ce moment de régression, elle scellera son propre destin. (Horkheimer et Adorno, 1983 : 15)

C'est donc en un sens au nom de la raison et des Lumières elles-mêmes qu'Adorno et Horkheimer entreprennent d'en faire la critique. Ce n'est pas d'un point de vue irrationaliste ou mystique qu'une telle critique salvatrice est entreprise, mais à partir de la raison elle-même, de manière immanente. D'ailleurs, on ne semble jamais vraiment pouvoir sortir de la raison chez Adorno et Horkheimer. Même l'opposition irrationaliste prend la forme d'une argumentation plus ou moins logique qui se soumet de facto aux impératifs de la raison. C'est pourquoi la Théorie critique insiste tant sur la dimension totalitaire et totalisante de la raison. Mais l'expression demeure chez eux volontairement ambiguë. C'est à la fois, une critique de la raison éclairée qui tend à réduire ou dissoudre toute forme de discours ou de jeux de langage alternatifs à l'aune de ses propres critères logico-mathématiques ${ }^{7}$. Mais c'est aussi l'affirmation d'une Raison qui habite depuis toujours l'ensemble des phénomènes de l'existence humaine, $y$ compris les phénomènes en apparence les plus irrationnels. Ces phénomènes, même sous la forme de mythes, sont déjà l'expression d'une certaine rationalité.

28 On voit bien là l'influence du kantisme, une influence peut-être plus grande que ce que les penseurs de la Théorie critique seraient prêts eux-mêmes à reconnaître. Il est clair cependant que la pensée kantienne est à situer du même côté que la leur, c'est-à-dire du côté du refus d'un réductionnisme de la raison à sa logique instrumentale. Le primat kantien de la raison pratique sur la raison théorique le montre bien. Chez Kant aussi, la raison se décline selon plusieurs modes qu'on ne saurait réduire les uns aux autres. Cela n'empêchera pas cependant la critique de la morale kantienne qui reste, aux yeux d'Adorno par exemple, trop prise dans la lignée de la raison théorico-scientifique avec l'idée de lois (entendue au sens que lui accorde la physique newtonienne), de pureté (selon une métaphore issue du champ de la chimie), ou le rejet de l'hétéronomie sensible qui condamne par le fait même la morale à hypostasier une raison désincarnée (Adorno, $2007:$ 302sq.).

29 Quoi qu'il en soit, l'entreprise de la Dialektik der Aufklärung doit se lire avant tout comme une auto-critique de la raison à la manière de la Première Critique kantienne (Assoun, 2012 : 38-39). Cette fois cependant, ce n'est pas de la Raison pure théorique et de l'avenir de la métaphysique dont il s'agit, mais de la raison dans ses multiples 
facettes, éthiques, esthétiques et critiques qu'il s'agit de préserver de la barbarie sociale et historique: "la Raison doit prendre conscience d'elle-même si les hommes ne doivent pas être trahis totalement ", écrivent Adorno et Horkheimer (Horkheimer et Adorno, $1983: 17)^{8}$. À l'instar de Kant, la Théorie critique s'articule ainsi autour d'un renouvellement de la pensée rationaliste. Comme l'écrit Paul-Laurent Assoun: «la Théorie critique confirmera le rationalisme tout en le rénovant. La récurrence du terme Raison n'est décidément pas fortuite: point de salut critique hors de l'affirmation décidée de la Raison selon le Sapere aude des Lumières » (Assoun, 2012 : 51). Il y a donc comme un fond d'Aufklärung dans la critique adornienne et hokheimerienne des Lumières. D'ailleurs, comme l'indique Rolf Wiggerhaus dans sa monumentale histoire de l'École de Francfort : « Horkheimer et Adorno ne désiraient pas jeter le bébé avec l'eau du bain, mais simplement démontrer l'ambiguïté des Lumières " (Wiggerhaus, 1993 : 314-15).

30 Plutôt qu'une critique unilatérale de celles-ci, la Théorie critique articule une tentative de préserver le potentiel authentiquement émancipateur des Lumières contre la forme pervertie qui en revêt les habits pour en détruire le potentiel libérateur même. Comme le suggère Wiggerhaus :

La DdA donnait l'impression de réunir de force deux concepts des Lumières : dans l'un, les Lumières poursuivaient un but, placer les hommes au rang de seigneurs, et une fois ce but atteint, elles faisaient briller sur la terre complètement acquise aux Lumières le jour du mal radical; dans l'autre, les Lumières visaient à apaiser cette revendication de domination et sa réalisation signifiait le renoncement à la puissance; en fin de compte, la première impression était que les Lumières se détruisent elles-mêmes et ne peuvent se sauver par elles-mêmes. Mais, au deuxième examen, on devinait au second plan la thèse jamais avouée : les fausses Lumières empêchent la victoire des vraies, victoire qui seule pourrait nous préserver des fatales conséquences des fausses Lumières. (Wiggerhaus, $1993: 320$ )

31 On serait ainsi confronté historiquement à deux versions des Lumières. La première, qui n'est pour l'École de Francfort qu'un travestissement de l'idéal des Lumières et qui trouve sa formulation paradigmatique dans les pensées de la raison instrumentale de Thomas Hobbes et Francis Bacon jusqu'au positivisme du $\mathrm{XX}^{\mathrm{e}}$ siècle, consiste à libérer l'homme par le biais d'une domination de la nature qui se retourne en définitive contre l'homme lui-même dont elles s'appliquent à dominer la nature sensible et irrationnelle. C'est finalement la nature même de l'homme dans ce qu'elle a d'irrationnel et l'homme lui-même qui sont tout aussi bien instrumentalisés à des fins opératoire et politique dans un monde où barbarie et progrès techniques se superposent l'un à l'autre de manière indifférenciée. L'idéal de ces Lumières travesties, c'est ce que la Théorie critique associe à la réalité de nos sociétés contemporaines, c'est-à-dire un monde où toute contingence et tout aspect irrationnel est administré ou réorienté, instrumentalisé à des finalités " productives ", c'est-à-dire à la destruction de la nature, du penser et de l'autonomie. C'est aussi ce monde totalement administré qui constitue l'obsolescence de l'homme et de sa responsabilité individuelle. «Ce dont il est question, comme l'écrit Ratté, c'est de la possibilité d'une hétéronomisation irréversible de l'homme qui serait déjà en marche, une hétéronomisation le déresponsabilisant toujours plus et le menant lentement mais sûrement vers une catastrophe environnementale planétaire " (Ratté, 2010:231). Le terme d'hétéronomisation est ici important, car c'est ce diagnostic de la Théorie critique qui rend manifeste, a contrario, ce qui constitue le cœur de leur reprise du projet kantien. 
32 Face au danger de l'hétéronomisation des individus, il s'agit de réaffirmer l'autonomie des sujets critiques, réflexifs, mais dont la réflexivité ne saurait se réduire à sa simple expression logico-mathématique. En fait, pour situer les deux concepts de Lumières qui s'opposent au sein de la Théorie critique, il faut parler d'une opposition en termes d'autonomie et d'autonomisation.

33 Les fausses Lumières travesties du monde administré qui finissent par se retourner contre les êtres mêmes qu'elles prétendaient libérer prennent en effet la forme d'une autonomisation de la raison: "L'homme ne se définit plus que comme une chose, comme élément de statistiques, en termes de succès ou d'échec. Ses critères sont l'autoconservation, la conformité - réussie ou manquée - à l'objectivité de sa fonction et aux modèles qui lui sont donnés " (Horkheimer et Adorno, 1983 : 44-45; 99-101). Plutôt que d'« être constamment à soi-même son propre législateur » comme le voulait les Lumières kantiennes selon Rudolf Eisler (Eisler, 2011 : 646)' ${ }^{9}$, l'individu moderne est soumis, pour l'École de Francfort à l'impératif hétéronomique de l'objectivité sociale qui en détermine la fonction et la conformité. Quelle place reste-t-il alors pour l'autonomie réflexive et la responsabilité individuelle?

34 De la Shoah aux expériences de Milgram, la soumission au devoir sous une forme hétéronomique, entraîne l'abandon du noyau constitutif des véritables Lumières. Des Lumières kantiennes, la Théorie critique reprend donc l'idée d'autonomie comme un moment absolument fondateur. «[L]a loi morale, affirme Kant, n'exprime pas autre chose que l'autonomie de la raison pratique pure, c'est-à-dire de la liberté, et cette autonomie est elle-même la condition formelle de toutes les maximes» (Kant, 2003 : 130). Ce passage cité par Horkheimer (Horkheimer, 1996:260) suffit à montrer à quel point l'autonomie constitue l'un des fondements les plus essentiels de l'Aufklärung y compris pour la Théorie critique (Adorno, $2001: 62$ ).

35 Ce qui n'empêche pas celle-ci de critiquer vertement la morale kantienne. Au contraire. Mais encore une fois, c'est comme si l'on assistait à une reformulation voire une radicalisation du projet kantien qui prend forme à la fois avec et contre Kant. Car dans l'autonomie fondée sur la raison pratique pure, Adorno et Horkheimer entrevoient le risque d'une autonomisation de cette raison face au sujet réfléchissant ou rationnel. C'est en définitive la raison à laquelle participe l'homme qui est autonome et non l'individu rationnel lui-même. Ce qui pour Kant ne fait pas de réelle différence constitue un problème central pour les penseurs de la Théorie critique. Dans la pureté de la raison pratique, Adorno aperçoit en effet la négation du sensible et voit là une dimension répressive propre au rigorisme kantien susceptible d'opposer à terme le devoir de la raison et la sensibilité morale qu'il s'agit pour la Théorie critique de préserver contre la pensée instrumentale (Adorno, 2007: 314sq.; Horkheimer et Adorno, 1983 : 92-94; Zima, $1974: 84$ ).

36 Malgré cette critique de Kant qui suggère que celui-ci tombe peut-être malgré lui du côté des Lumières répressives, l'Aufklärung de la Théorie critique ne saurait être comprise indépendamment de l'héritage kantien formulé autour des concepts d'autonomie et de réflexivité critique. "L'autonomie et l'unicité de l'individu cristallisent, en effet selon Horkheimer et Adorno, la résistance contre la puissance aveugle, oppressante de la totalité irrationnelle» (Horkheimer et Adorno, $1983: 262$ ). Contrairement à Kant cependant, cette autonomie doit prendre en compte non seulement l'autonomie réflexive de la raison face au sensible, mais l'autonomie du sujet sensible face à l'autorité d'une raison instrumentale autonomisée. On ne saurait 
prendre l'individu comme fin et jamais comme moyen sans prendre en compte sa dimension sensible. La souffrance, loin d'être uniquement une manifestation de la liberté s'élevant au-delà de l'inclination naturelle, doit au contraire constituer une balise de l'agir moral ${ }^{10}$. Si je peux vouloir souffrir pour affirmer mon autonomie et ma liberté comme sujet moral, je dois cependant refuser toute forme de souffrance imposée à un tiers au nom d'une fin, morale ou pas. C'est à la troisième personne que se décline l'incorporation de la responsabilité morale, d'où l'importance de la pitié et de l'empathie dont Adorno reproche justement à Kant de négliger l'importance (Adorno, 2007 : 315). En ce sens, il y a un certain rousseauisme aux Lumières et à la morale minimaliste de l'école de Francfort.

37 Mais il ne faudrait pas pour autant négliger l'importance de Kant, car tout ce que cette morale minimale cherche à préserver à l'heure de sa disparition, c'est le noyau même de l'éthique kantienne. C'est bien de la réification (Horkheimer et Adorno, $1983: 44$ ), du conformisme (Horkheimer, 1996: 270), «de la perte de son autonomie, de la désintégration de son Moi moral» (Zima, 1974:62) qu'il faut désormais préserver l'individu. Il s'agit d'opposer à la masse sérielle, mécanisée et instrumentalisée, c'est-àdire aux individus comme simples moyens, l'individu comme fin en soi, autonome et encore capable de penser et sentir de manière réflexive. Mais face à l'administration de la totalité ir-rationnelle et l'« érosion de l'autonomie » (Bronner, 2011 : 62) quasi totale, une telle opposition retombe souvent dans l'entreprise minimale de conservation, de préservation avec toute la dimension écologique que l'on peut associer à ces termes. Pour le dire autrement, c'est la biodiversité morale et intellectuelle qu'il s'agit de préserver de l'uniformisation d'une raison instrumentale insensible à la souffrance et la misère.

Mais au-delà de cette tâche de préservation qui prend un sens nouveau - mais clairement anticipé par Horkheimer et Adorno - à l'ère de la catastrophe écologique, la Théorie critique, malgré sa volonté, est demeurée incapable de formuler positivement le concept de ces Lumières véritables qui demeurent néanmoins le fondement rationnel de leur critique de la société moderne. Comme l'écrivent Stephen Eric Bronner (Bronner, 2011 : 62) et Wiggerhaus (Wiggerhaus, 1993 : 321), Horkheimer avait l'espoir d'articuler une doctrine dialectique positive des Lumières, lesquelles semblaient en effet avoir besoin d'être secourues et réappropriées par la Théorie critique et la praxis émancipatrice. Mais un tel projet n'a jamais été réellement mené à bien.

\section{Horkheimer ou les Lumières entre crépuscule et éclipse}

39 Cette volonté inaboutie est d'ailleurs palpable dans les travaux d'Horkheimer. Avec Dämmerung écrit pratiquement en exil à Zurich (sous le pseudonyme d'Heinrich Regius), on sent que l'espoir dans les Lumières incarnées par le mouvement ouvrier s'éteint lentement, mais sûrement. " Désabusée de l'espoir révolutionnaire, la Théorie critique semble se retourner vers un programme minimum de préservation de ce qui peut être encore sauvé de "l'autonomie de l'individu" (Assoun, 2012: 121). Mais un tel travail de préservation de l'autonomie et des Lumières est aussi fragile que la vie de l'individu sur lequel il repose. C'est pourquoi Horkheimer parle d'un crépuscule ou d'une éclipse de la raison (Horkheimer, 2004; Wiggerhaus, 1993: 331). Mais derrière un tel pessimisme, un espoir fragile point à l'horizon. Il se peut que l'éclipse des Lumières ne 
soit que momentanée. Comme l'écrit Wiggerhaus : «la pensée indépendante sauvait [pour Horkheimer] la "dernière trace de sens" " (Wiggerhaus, 1993: 334) non par une pratique politique à visée émancipatrice, mais en cherchant à réactiver les vestiges d'une raison substantielle dans l'histoire de la culture humaine. Encore une fois, contre une raison instrumentale et unidimensionnelle se définissant par son opposition abstraite au mythe, Horkheimer visait la réaffirmation du sens dans une raison substantielle et protéiforme. Or, une telle position est aussi à chercher du côté de Kant. Contre l'accusation de dirigisme de la raison (philosophique) telle que formulée par Habermas, contre l'idée selon laquelle le modèle kantien de la raison serait celui d'une raison strictement théorico-scientifique (Marcuse, Adorno), il faut en effet reconnaître la division des pouvoirs au sein de l'institution même de la raison. En affirmant encore une fois la primauté de la raison pratique et de la dignité humaine sur la raison instrumentale, Kant anticipe déjà la Théorie critique du moins en un sens large. Et Horkheimer lui-même semble reconnaître la dette de la Théorie critique envers Kant lorsqu'il écrit : «La société ne deviendra rationnelle que lorsque l'espoir kantien sera réalisé » (cité dans Zima, 1974 : 123). En attendant, ce qui s'affiche comme Lumières et raison n'en demeurent, à ses yeux, qu'une version pervertie et destructrice.

\section{Adorno et les apories de la Théorie critique}

40 La difficulté est cependant de penser les médiations sociales permettant la réalisation d'un tel espoir kantien dans un monde de plus en plus administré. On a vu que selon Zima, il y avait, suite à la faillite du mouvement ouvrier et son incorporation à la logique - qu'elle soit capitaliste et/ou totalitaire - des États modernes, une régression de la réflexion d'ordre stratégique, institutionnelle et politique (avec Hegel et Marx), à une position de repli sur la morale et l'individu autonome (avec Kant). On passe alors d'une logique offensive, voire révolutionnaire, à une entreprise défensive de préservations des possibilités réflexives et critiques constamment mises à mal par le déploiement de la logique instrumentale dans les diverses sphères - objectives et subjectives - de la vie sociale. Face à la faillite de la praxis émancipatrice, on assiste alors en quelque sorte avec la Théorie critique à un repli volontaire sur le théorique (Adorno, $2007:$ 11).

41 Ce qu'on pourrait par conséquent considérer comme les apories pratiques de la Théorie critique donnent lieu paradoxalement à une position que les penseurs de l'École de Francfort reprochaient eux-mêmes aux Lumières kantiennes. Dans sa leçon du 9 juin 1959 sur la Critique de la raison pure, Adorno articule une critique que nous avons déjà rencontrée chez Horkheimer et Marcuse : il n'y a pas de véritable liaison entre les Lumières et l'action pratique ou politique (Adorno, 2001:62). C'est « comme savant» (Kant, 1985 : 211) seulement, et non en tant qu'individu ou comme citoyen que je peux exercer ma liberté de conscience. Cette «division du travail» (Adorno, 2001: 63) qui délimite et confine les Lumières à un champ, celui de la conscience ou de l'expertise est vue par Adorno comme un repli par rapport au potentiel émancipateur des Lumières. Seul l'être humain pris en son sens purement théorique - et désincarné est libre d'être éclairé en un sens radical (Adorno, $2001: 63$ ).

42 Contre cette vision abstraite de l'individu éclairé, nous avons vu tout au long de cette présentation que la Théorie critique opérait en quelque sorte une radicalisation des principes des Lumières kantiennes. Ce n'est plus simplement le savant qui doit faire un 
usage public de sa raison, mais c'est l'individu, l'être humain concret en tant que tel. Mais en quoi consiste au juste cet usage public de la raison humaine dans le cadre d'un monde où « les fins les plus dignes de la raison, celles qui rendent l'homme libre et responsable» et que la Théorie critique cherche justement à préserver «sont harnachées par une conceptualisation qui ajourne à l'infini la possibilité que le sujet les assument [ces fins] comme projet » (Ratté, $2010: 226)$ ?

La Théorie critique semble se limiter, comme nous l'avons vu, à la tentative de préserver ou maintenir en vie la possibilité de l'humanité même, des Lumières authentiques en se repliant sur la théorie, l'esprit critique, la réflexion sans cesse mise à mal, mais seuls fondements possibles d'une liberté et d'une responsabilité adéquate au réel. Ce faisant cependant, on retombe en quelque sorte du côté de l'abstraction que les auteurs de la première génération de l'École de Francfort reprochaient eux-mêmes à Kant. Pas plus que Kant, ils ne semblent en mesure d'articuler le projet des Lumières avec une action politique. Et à ce titre, il faut noter que malgré sa critique des Lumières kantiennes, ce qu'Adorno retient de l'héritage de celles-ci est avant tout cette réflexivité critique du dogmatisme qui constitue peut-être le cœur non seulement d'une éventuelle Aufklärung francfortoise, mais de tout le projet même d'une Théorie critique (Adorno, 2001 :59). Là où survivent les Lumières kantiennes, là où leur valeur est maintenue, pour Adorno, c'est dans l'approche critique, le refus du dogmatisme, la reconnaissance des limites de l'entendement et la possibilité d'une nouvelle méthode (micrologique) d'appréhension du réel. En ce sens, les Lumières kantiennes sont sinon immortelles du moins préservées par la pensée critique comme théorie. Mais le passage de cette réflexivité critique, de la théorie à la pratique, reste largement à penser et à faire.

\section{BIBLIOGRAPHIE}

Adorno, Th. W., 2001, Kant's critique of Pure Reason, Standford : Standford University Press.

Adorno, Th. W., 2007, Dialectique négative, Paris : Payot.

Adorno, Th. W., 2008, Minima Moralia, Paris: Payot.

Assoun, P.-L., 2012, L'École de Francfort, Paris : PUF.

Baynes, K., 2004, « The Transcendental Turn : Habermas's “Kantian Pragmatism” » in Rush F. (dir.), The Cambridge Companion to Critical Theory, Cambridge : Cambridge University Press, 2004, p. 194-218.

Benoist, J.-M., 1970, Marx est mort, Paris : Gallimard.

Bronner, S. E., 2011, Critical Theory - A Very Short Introduction, Oxford : Oxford University Press.

Castoriadis, C., 1999, L’Institution imaginaire de la société, Paris : Seuil.

Eisler, R., 2011, Kant-Lexikon (2 vol.), Paris : Gallimard.

Habermas, J., 1986, L'Espace public, Paris : Payot. 
Habermas, J., 1987, Profils philosophiques et politiques, Paris : Gallimard.

Habermas, J., 1992, De l'éthique de la discussion, Paris : Cerf.

Habermas, J., 1997, Morale et communication, Paris : Cerf.

Horkheimer, M., 1980, Les Débuts de la philosophie bourgeoise de l'histoire, Paris : Payot.

Horkheimer, M., 1996, Théorie traditionnelle et théorie critique, Paris : Gallimard.

Horkheimer, M., 2004, Eclipse of Reason, London: Continuum.

Horkheimer, M., Adorno, Th. W., 1983, La Dialectique de la raison, Paris : Gallimard.

Kant, E., 1985, Cuvres philosophiques, tome II, Paris : Gallimard/La Pléiade.

Kant, E., 2003, Critique de la raison pratique, Paris : GF-Flammarion.

Marcuse, H., 1963, Le marxisme soviétique, Paris : Gallimard.

Marcuse, H., 1968, Raison et révolution, Paris : Éditions de minuit.

Marcuse, H., 1971a, Pour une théorie critique de la société, Paris : Denoël/Gonthier.

Marcuse, H., 1971b, Eros et civilisation suivi de La notion de progrès à la lumière de la psychanalyse, Paris : Éditions de Minuit.

Marcuse, H., 1991, L'Ontologie de Hegel et la théorie de l'historicité, Paris : Gallimard.

Marx, K., 2009, Le Capital Livre I, Paris : PUF.

Piché, Cl., 1995, Kant et ses épigones, Paris : Vrin.

Ratté, M., 2010, «Réflexions cursives pour l'articulation de la Théorie critique et de la phénoménologie », in Coutu B. (dir.), Actualité de la théorie critique, Montréal : Éditions libres du carré rouge, 2010, p.225-46.

Schmidt, A., 1973, Emanzipatorische Sinnlichkeit - Ludwig Feuerbachs anthropologischer Materialismus, München: Reihe Hanser.

Wiggerhaus, R., 1993, L'École de Francfort, Paris : PUF.

Zima, P., 1974, L'École de Francfort, Paris : Éditions universitaires.

\section{NOTES}

1. Voir (Kant, $1985: 211)$.

2. Allant dans le même sens, Adorno affirmera qu'il n'y eut à proprement parler pas de Lumières allemandes, mais seulement une théologie éclairée (Adorno, 2001 : 58).

3. On retrouve le même reproche chez Habermas ou Adorno pour lesquels Kant adopte une approche beaucoup trop subjectiviste de l'éthique et l'esthétique (Adorno, 2001: 62 ; Baynes, $2004: 195,197$; Ratté, $2010: 227$ ). Il faudrait cependant se demander si la première génération de l'école de Francfort ne tombe pas dans une nouvelle version du même travers en cherchant à préserver l'autonomie individuelle et la pensée critique de l'intégration à la totalité sociale.

4. Ce titre est repris de Jean-Marie Benoist (Benoist, 1970).

5. On pense bien sûr ici à Marcuse (Zima, 1974 : 20), mais aussi à Horkheimer : « Horkheimer se considérait comme un représentant de la théorie marxiste - dans le sens où la position qu'il occupait se trouvait dans le prolongement d'une ligne qui partait de Kant et des philosophes français des Lumières et passait par Hegel et Marx » (Wiggerhaus, 1993 : 52). 
6. Même Adorno qui est peut-être le plus critique de la raison éclairée continue à articuler sa réflexion comme une ultime tentative de sauvegarder la raison contre elle-même au moment de sa chute. « Adorno a encore un projet pour la raison » (Ratté, $2010: 226$ ).

7. Cf. (Horkheimer et Adorno, $1983: 41)$ : « Même l'insoluble et l'irrationnel n'échappent pas aux théorèmes mathématiques. En identifiant d'avance à la vérité le monde mathématisé et entièrement organisé par le penser, la Raison se croit à l'abri du mythe. Elle assimile le penser aux mathématiques ».

8. Sur l'historicisation de la critique de la raison par elle-même, voir (Assoun, 2012 : 115-16).

9. Adorno fera l'éloge du Kant Lexikon de Eisler dans son traitement des Lumières kantiennes, cf. Adorno, Kant's Critique of Pure Reason, p.60.

10. En ce sens il semble bien que ce soit avec Ludwig Feuerbach que les penseurs de la Théorie critique entendent réformer le moralisme kantien. Voir à ce propos le bel ouvrage d'Alfred Schmidt, étudiant d'Adorno et Horkheimer (Schmidt, 1973).

\section{RÉSUMÉS}

Cet article propose un panorama du rapport ambivalent qu'adoptent les principaux auteurs de l'École de Francfort vis-à-vis de la conception kantienne des Lumières. En passant par Habermas, Marcuse, Horkheimer et Adorno, nous nous proposons de montrer en quoi c'est à la fois avec et contre Kant que s'articule la Théorie critique. Nous tâcherons de montrer par ailleurs en quoi la première génération de l'École de Francfort reconduit en un sens l'aporie qu'elle soulignait déjà au sein de la posture kantienne quant à l'articulation entre théorie et pratique émancipatrice.

\section{INDEX}

Mots-clés : Autonomie, Dialectique des Lumières, École de Francfort, Emmanuel Kant, Théorie critique

\section{AUTEUR}

\section{EMMANUEL CHAPUT}

Doctorant en philosophie, professeur à l'Université d'Ottawa (Canada). 